\title{
A New 3D Sensor System for Mobile Robots Based on Moire and Stereo Vision Technique
}

\author{
Hyunki Lee and Hyungsuck Cho \\ Department of Mechanical Engineering, \\ Korea Advanced Institute of Science and Technology \\ Daejeon, South Korea \\ \{hklee and hscho\}@lca.kaist.ac.kr
}

\author{
Minyoung Kim \\ Research Team of Vision System \\ Koyung Technology Co. Ltd. \\ Seoul, South Korea \\ mykim@1ca.kaist.ac.kr
}

\begin{abstract}
Intelligent autonomous mobile robots must be able to sense and recognize 3D environment for navigation and task execution, where they live or work. To achieve this, a variety of techniques have been developed for the determination of the 3D scene geometric information such as stereo vision, laser structured light, laser range finder and so on. But these methods have many limitations such as susceptibility to noise, low speed in 3D scene etc. To overcome these limitations we introduce a new sensing algorithm, which is based on the moire technique and stereo vision. To verify the efficiency and accuracy of our algorithm, a series of experimental tests is performed.
\end{abstract}

\section{Index Terms - Active Vision, Moire Technique, Stereo Vision, Sensor Fusion}

\section{INTRODUCTION}

Nowadays a major research issue of mobile robots is the development of environment sensing and recognition system for navigation and task execution. A variety of machine vision techniques [1-4] have been conducted for the determination of 3D scene geometric information from 2D images. Although binocular and trinocular vision [13] sensors among them have been widely used as representative ones of passive sensor system, they still suffer from sudden changes of image intensity due to the illumination noise, insufficient feature information on environment composed of plain surfaces and correspondence problem between multiple images. These reasons have made most mobile robot researches on 3D environment reconstruction using visual sensors deal with just straight line edge and corner as interesting features $[2,3,5,6]$. However, these features are saliently observed only in well arranged, structured environments with simple polygonal objects or polygon-textured surfaces. This information may be not sufficient to describe the whole structure of 3D space [7].

In many approaches for mobile robot applications, laser sensor has been frequently used for detail sensing because of the robustness to the nature of the navigation environment and the easy extraction of feature information of interest $[9,10]$. The LRF (laser range finder) measuring the time-of-flight between the sensor and object [11,12] is one example of such laser sensor. The disadvantage is that it maintains the same resolution even at a short distance and need still high cost with high power consumption, scanning process and heavy weight. To decrease the sensing time without degradation of the sensor resolution, it is necessary to develop a new visual sensor system different from the sensors mentioned above.
To achieve this purpose effectively, we introduce a new sensing algorithm based on the moire technique. [14] The technique is well known for its quick, fast and accurate 3D measurement. It is based on active vision sensor system, so the measurement is robust to illumination noises and consequently can acquire the accurate result. In addition to this sensing characteristic, the sensing time is very short. Due to this advantage, these days, it has been used for many applications to measure the 3D shape. These favorable characteristics make it possible to adopt the moire technique as a mobile robot sensing system. But there is one limitation: The general moire technique cannot measure the absolute 3D information because of $2 \pi$-ambiguity. To overcome this limitation we apply a binocular camera system to the original moire technique. It is shown that by combining these two techniques we can obtain the absolute value of the 3D scene geometric information with satisfactory accuracy.

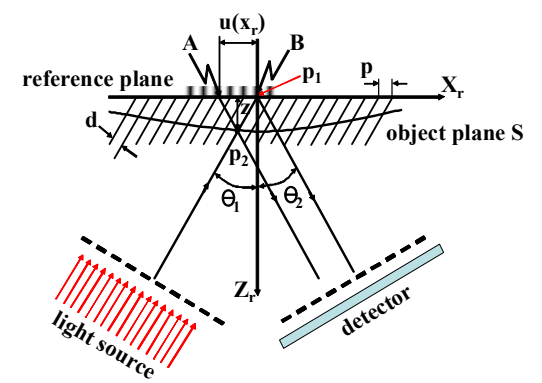

Fig. 1 The fringe projection geometry of projection moiré

\section{Moire TeChNiQue}

\section{A. Projection Moire Technique}

Fig. 1 shows the principle of the projection moire technique. Here, to make a problem simple, we assume that the light wave projected to the object is a plane wave with oblique angle $\theta_{l}$. The target of this method is to find the depth value $z$ of point $\mathrm{p}_{2}$ on an object surface. If we can measure the distance value $u\left(x_{r}\right)$, the depth of $\mathrm{p}_{2}$ can be estimated by using the geometry of the system. The distance value $u\left(x_{r}\right)$ can be estimated from modulation function, $\psi\left(x_{r}\right)$, in (3). The $\psi\left(x_{r}\right)$ values founded by general moire technique and projection moire technique are found to be identical. [15]

Now we will discuss the geometric method to find the phase modulation value $\psi\left(x_{r}\right)$. If there is no object, the 
intensity value of point $\mathrm{A}$ on the reference plane can be detected in the camera at a given pixel. If there is an object plane (S), the intensity value of point $B$ in the camera is detected at the given pixel. To do this first we detect the A value at given pixel, and then put the object, we detect the B value at given pixel. The intensity value difference between points $\mathrm{A}$ and $\mathrm{B}$ indicates the distance, $u\left(x_{r}\right)$, value. Then we can estimate the depth value of $z$.

$$
u\left(x_{r}\right)=z\left(\tan \theta_{1}+\tan \theta_{2}\right)
$$

After detecting the depth value in $\mathrm{Y}_{\mathrm{r}}$, then choosing another row line and adopting the algorithm again. In (1), $\theta_{1}$ and $\theta_{2}$ have been already given based on the hardware architecture as shown in Fig. 1. [15] However, actually making a plane wave of the illumination for wide environment is very difficult. A CCD camera which is assumed to be projection model is used as detector as shown in Fig. 2. [15]

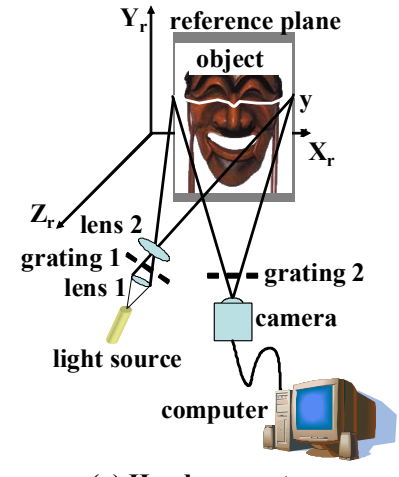

(a) Hardware setup

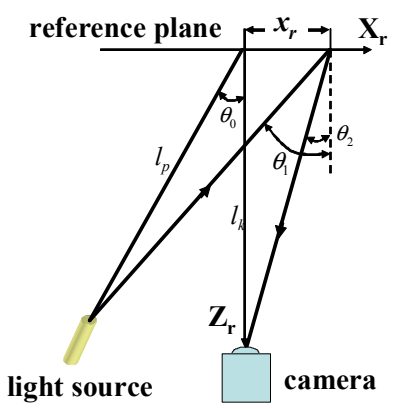

(b) Fringe projection geometry
Fig. 2 Hardware setup and geometry of projection moire

The projection pattern which looks like a cone shape is projected to the object by using a lens system and the camera has a perspective projection characteristic. If we choose one arbitrary line, $y_{r}$, in reference frame, along the white line in the figure the $\mathrm{Y}_{\mathrm{r}}$-axis direction fringe pattern is defined. Here, to adopt the moire technique, we select one surface and $y_{r}$ line in the reference plane, inclusive of the white line. Then, we can obtain the geometric relationship between the camera and light source as shown in Fig. 2(b).

The projection moire technique has a good measurement resolution, but it has a complex hardware system as shown in Fig. 2. Here, the pitch of the gratings of projection moire technique is fixed. [16, 17]

To overcome this inconvenience Phase Modulate Profilometer(PMP) method is generally used as shown in Fig. 3. In this system, as turning on and off the laser diode periodically and changing the angular velocity of polygon mirror, we can get a variety of different patterns, which then can be used for various environments. Photo diode is to detect the on and off the laser diode for controlling the on and off time.

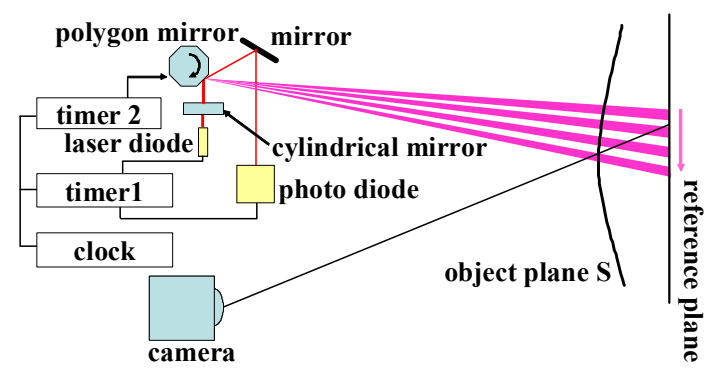

Fig. 3 Hardware system and sensing principle of PMP

B. Limitation of the Projection Moire Technique

The projection moire technique, however, has limitation to the phase difference estimation.

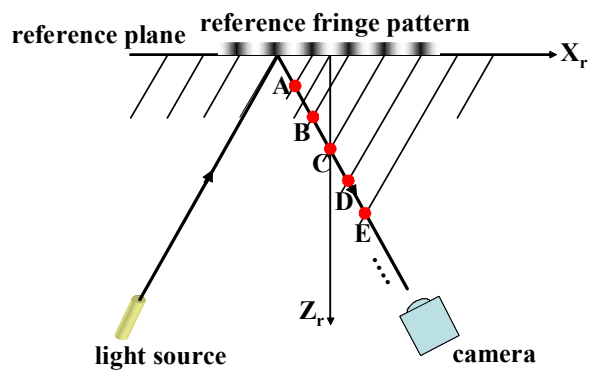

Fig. $42 \pi$-ambiguity of projection moiré technique

For example, as shown in Fig. 4 the lowest intensity value is measured at a given pixel in the camera. In this case, points A, B, C, D, E,... in Fig. 4 become candidate points for depth measurement, according to projection moire technique. Because projection fringe pattern appears periodically, this kind of phenomenon occurs, which causes ambiguity for finding a depth. We call this $2 \pi$-ambiguity. If the phase difference between phase of reference plane and that of point $\mathrm{A}$ is $\varphi$, the phase of candidate points $\mathrm{B}, \mathrm{C}, \mathrm{D}, \mathrm{E}, \ldots$ can be written as follows :

$$
\psi\left(x_{r}\right)=2 \pi N+\varphi \quad \text { for } \quad N=0, \pm 1, \pm 2, \pm 3, \ldots
$$

where $\psi\left(x_{r}\right)$ is the modulation function, and $N$ is unknown. Here, $N$ expresses the $2 \pi$-ambiguity.

\section{Proposed Algorithm For 3D Sensing}

\section{A. Architecture of the proposed algorithm}

As shown in Fig. 5(a), the proposed sensor system consists of two cameras and one multi-stripe laser projector. In the proposed algorithm employing two cameras we can make two phase modulation equations as follows:

$$
\begin{array}{ll}
\psi_{L}\left(x_{r}\right)=2 \pi N_{L}+\varphi_{L} & \text { for } N_{L}=0, \pm 1, \pm 2, \pm 3, \ldots \\
\psi_{R}\left(x_{r}\right)=2 \pi N_{R}+\varphi_{R} & \text { for } N_{R}=0, \pm 1, \pm 2, \pm 3, \ldots
\end{array}
$$

where $\psi_{L}\left(x_{r}\right)$ and $\psi_{R}\left(x_{r}\right)$ are the modulation function at a given point, $\varphi_{L}$ and $\varphi_{R}$ are the smallest phase shifting value among candidate points at given point in the left and right camera respectively. Here $\varphi_{L}$ and $\varphi_{R}$ can be measured by the moire 
technique and the depth value $z$ in Fig. 2 which is estimated by $\psi_{L}\left(x_{r}\right)$ and $\psi_{R}\left(x_{r}\right)$ must be identical. Then, in (3) if we find the integer $N_{L}$ and $N_{R}$, respectively with measured $\varphi_{L}$ and $\varphi_{R}$, we can solve the (1) and therefore can overcome the $2 \pi$-ambiguity problem.

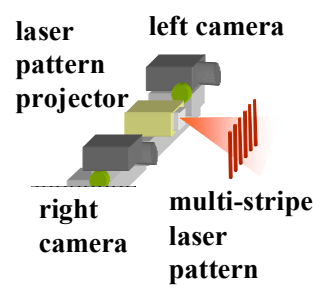

(a) Hardware setup for the proposed algorithm

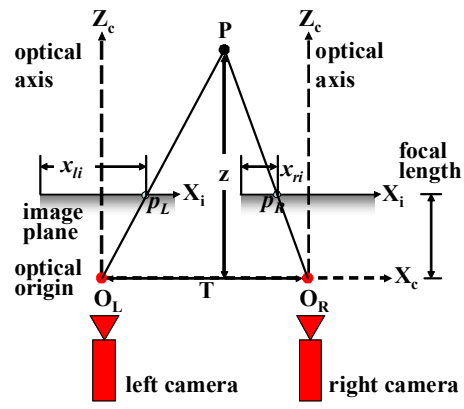

(b) Special stereo vision architecture
Fig. 5 Architecture of proposed algorithm

\section{B. Stereo Vision}

Fig. 5(b) shows the arrangement of a simple stereo vision. Here, two optical axes of the camera are parallel and two cameras' intrinsic parameters are identical. If it is assumed that the $x_{l i}$ in the left intensity image and $x_{r i}$ in the right intensity image are the correspondence points for point $\mathrm{P}$, then depth value of $\mathrm{P}$ can be estimated from

$$
z=\frac{T f}{x_{l i}-x_{r i}} \text {. }
$$

where $i$ means the $\mathrm{i}^{\text {th }}$ corresponding point at given scan line, $T$ is the distance between two cameras and $f$ is the focal length of the camera. The intrinsic parameters can be found by camera calibration. [19] We call the denominator of the (4) disparity, $d$, which is given by

$$
d=x_{l i}-x_{r i}
$$

There are several constraints of the stereo vision.

$$
\begin{aligned}
& \text { Constraint } 1(\mathrm{C} 1): 0 \leq x_{l i}-x_{r i} \\
& \text { Constraint } 2(\mathrm{C} 2): x_{l i}<x_{l j} \text { and } x_{r i}<x_{r j}, 1 \leq i<j \\
& \text { Constraint } 3(\mathrm{C} 3): x_{l i+1}=x_{l i}+1 \text { or } x_{r i+1}=x_{r i}+1
\end{aligned}
$$

The $\mathrm{C} 1$ indicates the characteristic of the positive disparity, and the $\mathrm{C} 2$ means that each correspondence point must be in order. The $\mathrm{C} 3$ deals with the occlusion.

\section{The Proposed Sensing Algorithm}

The schematic diagram of the hardware system which we have discussed in section III. $A$ is shown in more detail in Fig. 6(a). The world coordinate is located at the center of the left camera and the center point of pattern projector is located at $P_{1}$. As shown in the figure, the reference fringe pattern is generated by the pattern projector. If an object is located in front of the reference plane, the slightly changed fringe pattern images in the left and right image can be acquired as shown in
Fig. 6(a). Here, we choose in the same row in the left and right image: a-a' in the left image and b-b' in the right image, which is called the epipolar line for each other.

The first step is to search the candidate points for depth measurement based on the moire technique. In this step the pattern projector and right camera are used. We choose one fringe edge pixel at given scan line b-b'. Then, according to geometric relationship we can find the candidate points by using moire technique. For example, as shown in Fig. 6(a), we can select one arbitrary point, $\mathrm{R}_{1}$, which is located on the right side fringe pixel, and then can find the line equation of $\ell_{R_{1}}$ by using camera equation. Here, $\ell_{R_{1}}$ is the length of the line $O_{R} A$. In general $3 \mathrm{D}$ case, to express the line equation is very difficult. Therefore, the plane which consists of the right optical origin, scan line and given reference pattern can be obtained. In this case, the equation for $\ell_{R_{1}}$ is simply estimated by

$$
z=\frac{M_{R}(1,1)}{x_{R_{1}}-M_{R}(1,3)} x+\frac{M_{R}(1,4)}{x_{R_{1}}-M_{R}(1,3)}
$$

where $x_{R_{1}}$ is the $\mathrm{X}$-axis coordinate of the pixel $\mathrm{R}_{1}$ in the right image plane and $M_{R}(\mathrm{i}, \mathrm{j})$ is the element of $\mathrm{i}^{\text {th }}$ row and $\mathrm{j}^{\text {th }}$ column of the right camera projection matrix $M_{R}$. In this way, we can derive the simple line equation $\ell_{R_{\mathrm{I}}}$ independent of the $\mathrm{Y}$-coordinate. Then we try to find the candidate points for depth measurement by using moiré technique: The candidate points are defined as the intersection points of two lines; one line of which is line $\ell_{R_{1}}$ and the other of which connects $\mathrm{P}_{1}$ and fringes which share the same fringe edge as $R_{1}$ 's in the reference fringe pattern. The candidate points obtained in this manner (A, B, C, D, ...) are shown in Fig. 6(a).

The second step is based on stereo vision to find the depth value by searching the point corresponding to a given point in the right image. We project these points into the image plane of left camera. Fig. 6(b) shows this process briefly. We projects the candidates points $\mathrm{A}, \mathrm{B}, \mathrm{C}, \mathrm{D}, \cdots$ to the image plane of left camera. Then we can get the corresponding projected points $\alpha, \beta, \gamma$. In the case of point $\mathrm{D}$, the projected point is out of the image plane of left camera, so the point $\mathrm{D}$ is discarded. The left camera projection matrix is used for this process, whose equation can be written as : [18]

$$
S\left[\begin{array}{c}
x_{L} \\
y_{L} \\
1
\end{array}\right]=M_{L}\left[\begin{array}{c}
X \\
Y \\
Z \\
1
\end{array}\right]
$$

where $s$ is scale factor, $X, Y$ and $Z$ are the coordinates of the candidate points, $(\mathrm{A}, \mathrm{B}, \mathrm{C}, \mathrm{D}, \ldots)$ in the world coordinates, $x_{L}$ and $y_{L}$ are the image coordinates in the left camera and $M_{L}$ is a $3 \times 4$ camera projection matrix of left camera. 


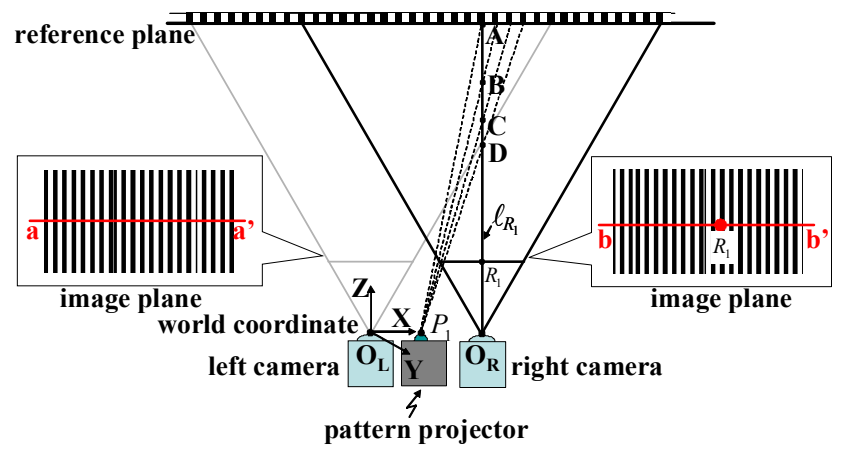

(a) First step based on the moiré technique

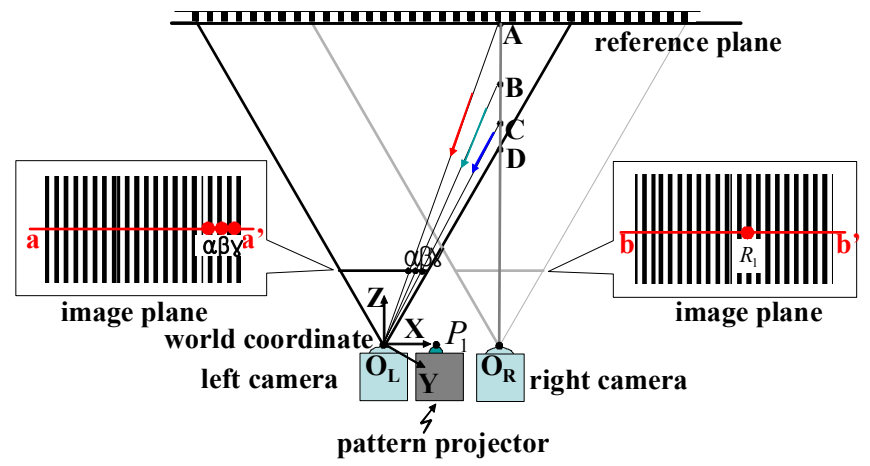

(b) Second step based on the stereo vision technique

Fig. 6 The proposed algorithm based on the moiré technique and stereo vision

After projecting all candidate points onto the left image, we compare the intensity value of the projected points in the left image with that of point $\mathrm{R}_{1}$ in the right image. According to the stereo vision principle, the corresponding points should have the same intensity value: if point $R_{1}$ is located at the left side edge pixel of the fringe, so is the correspondence point; if $\mathrm{R}_{1}$ is the right side edge pixel of the fringe, so is the correspondence point. In Fig. 6(b) the point $\alpha$ has the same intensity value as that of the given point $R_{1}$ and is also located in the same side edge pixel of the fringe as $R_{1}$. On the contrary, the other two points $\beta$ and $\gamma$ have different intensity values from that of the given point $R_{1}$ and are not located in the same side edge pixel of the fringe as $\mathrm{R}_{1}$. According to this result, we know that pixel $R_{1}$ in the right image and $\alpha$ in the left image are corresponding to each other. By using the $\mathrm{X}_{\mathrm{i}^{-}}$ coordinate of pixels $\mathrm{R}_{1}$ and $\alpha$ in the image coordinate, we can estimate the depth value of point A from (4).

\section{EXPERIMENTS For ENVIRONMENT SENSING}

\section{A. Hardware Setup for Experiments}

The experimental system consists of a laser pattern projector and two cameras as shown in Fig. 7.

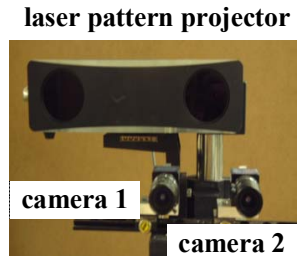

(a) Hardware setup for experiments

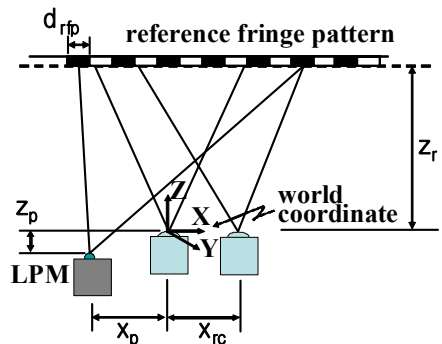

(b) Schematic diagram of the hardware setup

Fig. 7 Hardware setup for experiments

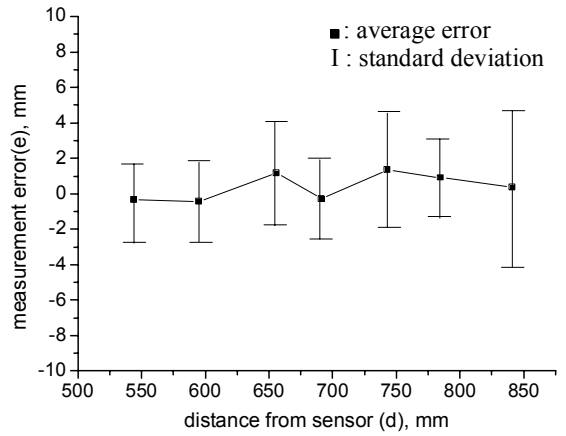

Fig. 8 Depth measurement using the proposed sensor

We put the world coordinates at the optical center of left camera and the optical axis of the right camera parallel to the left camera. The distance between two cameras, $x_{r c}$, is $13 \mathrm{~mm}$. The distance between two cameras is decided by experiments. The reference plane is located, $z_{r}$, at $550 \mathrm{~mm}$ in front of the sensor system as shown in Fig. 7(b). First, we calibrate the intrinsic parameter of the left and right cameras as follows by using Zhang's algorithm; [19]

$$
M_{\text {int }}=\left[\begin{array}{ccc}
\alpha & -\alpha \cot \theta & u_{0} \\
0 & \frac{\beta}{\sin \theta} & v_{0} \\
0 & 0 & 1
\end{array}\right]=\left[\begin{array}{ccc}
1067.03685 & 0 & 300.07963 \\
0 & 1071.10087 & 230.78618 \\
0 & 0 & 1
\end{array}\right]
$$

where $\alpha$ is $k f, \beta$ is $\ell f, k$ and $\ell$ are the horizontal and vertical scale parameters of the CCD unit, respectively, $f$ is focal length of the camera, $\theta$ is the skew angle of the CCD cell and $u_{0}$ and $v_{0}$ are the $\mathrm{X}_{\mathrm{i}^{-}}$and $\mathrm{Y}_{\mathrm{i}^{-}}$coordinates of the center point of the image plane, respectively. [18]

\section{B. Experiments on objects with various shapes}

To evaluate the performance of the sensor system, it is necessary to examine a simple object first. For this purpose, a flat plane is placed in front of the sensor system, and a laser fringe pattern is projected on it. With variation of the distance from the sensor to this plane, $d$, the measurement tests were performed, and the experimental results are summarized in Fig. 8. The results represent the accuracy of the sensor system and its error characteristics according to the variations of the distance. The error standard deviation tends to increase with the object distance. It is related to the stereo vision principle. 
If the measurement distance becomes large, the disparity value becomes small and then the measurement error becomes large.

TABLE I

MEASUREMENT RESULTS WITH DIFFERENT OBJECTS (UNIT : $\mathbf{m m}$ )

\begin{tabular}{|c|c|c|c|}
\hline & $\begin{array}{c}\text { real } \\
\text { value }\end{array}$ & moire technique & $\begin{array}{c}\text { proposed } \\
\text { algorithm }\end{array}$ \\
\hline thickness of the object 1 & 50 & 54 & 55 \\
\hline $\begin{array}{c}\text { distance between the front } \\
\text { surface of object 2 and reference } \\
\text { plane }\end{array}$ & 140 & 38 & 144 \\
\hline radius of the object 3 & 95 & 25 & 93 \\
\hline
\end{tabular}

Next, we performed measurement tests on three objects composed of square block and curved line as shown in Figs. 9, 10, and 11. A simple square and curved object were used in experiments. When each object is placed in front of the plane, a laser fringe pattern with $7 \mathrm{~cm}$ width was projected on it, and the $3 \mathrm{D}$ information extraction was performed by the proposed algorithm. In Figs. 9(d), 10(d) and 11(d), the acquired 3D point data on each object is represented with the same order of it shown in Figs. 9(a), 10(a) and 11(a). The experimental results show that obtained by the proposed sensor system measure the objects with plain surface, and the measured data represents them well. Table 1 shows the shape measurement results on the objects through a surface fitting method based on the least-square-error minimization. From these results we can know that the measurements values are very close to those of the real objects' data.

\section{Result Comparison between Typical Moire Technique and Proposed Algorithm}

In section III. $A$, it was pointed out that our proposed algorithm can overcome the $2 \pi$-ambiguity of the typical moire technique. To verify this we compare the result of moire technique with the result of our proposed algorithm. In Fig. 9(a), we put a simple box in front of the reference plane. The box size is $80 \times 210 \times 50 \mathrm{~mm}$. As we can see in Fig. 9(c) and (d), the result is almost identical between the moire method and proposed algorithm because the thickness value of the object 1 is less than that of the $2 \pi$-ambiguity. However, the $2 \pi$-ambiguity phenomena can be seen in Fig. 10 (c) from the result of typical moire method. Next, we put the box, where its dimension is $222 \times 400 \times 80 \mathrm{~mm}, 60 \mathrm{~mm}$ in front of the reference plane, so the distance between the reference plane and the front surface of the object 2 becomes $140 \mathrm{~mm}$. In Fig. 10(c), the measurement value, $38 \mathrm{~mm}$, is different from the real value. However, the results in a value closer as shown in Fig. 10(d) imply that the proposed algorithm can overcome the $2 \pi$ ambiguity. Likewise, the experimental results shown in Fig. 11(c) and (d) also support that that our proposed one doesn't suffer from the $2 \pi$-ambiguity.

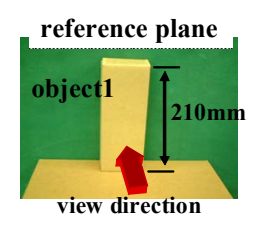

(a) object for experiment

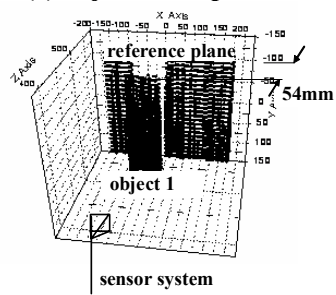

(c) result of moire technique

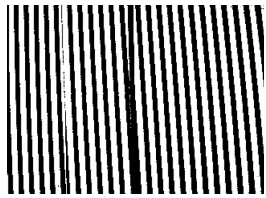

(b) right fringe pattern image

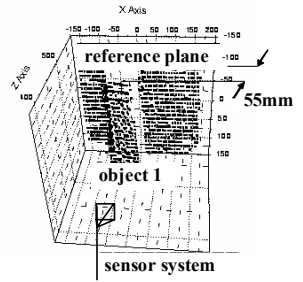

(d) result of proposed algorithm
Fig. 9 Experimental result for environment 1

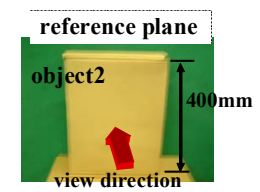

(a) object for experiment

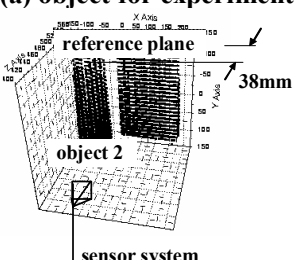

(c) result of moire technique

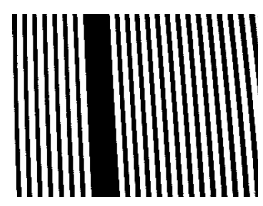

(b) right fringe pattern image

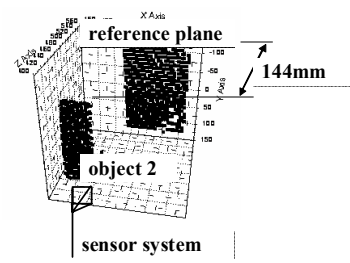

(d) result of proposed algorithm
Fig. 10 Experimental result for environment 2

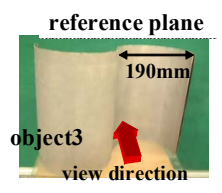

(a) object for experiment

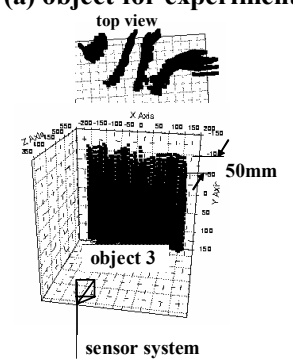

(c) result of moire technique

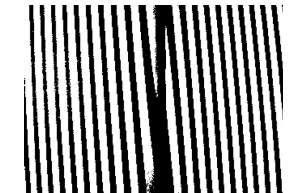

(b) right fringe pattern image

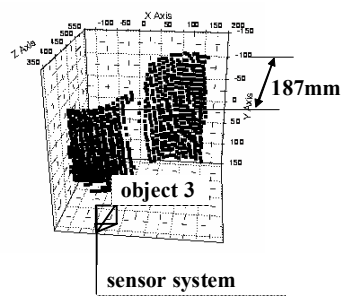

(d) result of proposed algorithm
Fig. 11 Experimental result for environment 3

\section{Parameter Analysis}

The width of the fringe pattern is one of the important factors for sensor resolution in X-axis. Now, we try to check the effect of the width of fringe pattern by comparing the real value of the objects and measured one. For experiments, we fix the distance between two cameras, $13 \mathrm{~mm}$, and varied the fringe pattern width from $40 \mathrm{~mm}$ to $130 \mathrm{~mm}$. In Table 2, the 
measured values of the objects seen to vary according to the width of the fringe pattern. It is related to the measurement resolution in $\mathrm{X}$-direction. When the fringe pattern with small fringe width is projected, the measurement width of the object is similar to that of the real objects. However, in the case of large fringe width, the error value of the measurement width becomes large. This result is based on the sampling problem along $\mathrm{X}$-axis.

TABLE 2

MEASUREMENT RESULTS WITH DIFFERENT FRINGE WIDTHS

\begin{tabular}{|c|c|c|c|c|c|}
\hline \multirow{2}{*}{ (UNIT : $\mathbf{m m}$ ) } & \multirow{5}{*}{$\begin{array}{c}\text { measurement result with } \\
\text { different }\end{array}$} & \multicolumn{4}{|c|}{ fringe width } \\
\cline { 3 - 6 } & value & 40 & 70 & 100 & 130 \\
\hline width of the object 1 & 80 & 81 & 71 & 69 & 66 \\
\hline width of the object 2 & 94 & 95 & 98 & 87 & 77 \\
\hline diameter of the cylindrical object & 70 & 70 & 72 & 59 & 64 \\
\hline
\end{tabular}

Next, we check the effect of the distance between two cameras. We fixed the width of the fringe pattern, and changed the distance between two cameras from $7 \mathrm{~mm}$ to $25 \mathrm{~mm}$. As shown in Table 3, it is found that the distance between two cameras must be near $13 \mathrm{~mm}$. When the distance between two cameras is short, no difference is seen between two acquired fringe images causing measurement errors. So the error becomes a little large. On the contrary, it became too long so that the distance between corresponding points becomes long and it causes errors. Therefore we need to choose a proper distance between two cameras.

\section{TABLE 3}

Measurement Results with Different Camera Position

\begin{tabular}{|c|c|c|c|c|c|}
\hline (UNIT $:$ mm) & real & \multicolumn{4}{|c|}{ distance between two camera } \\
\cline { 3 - 6 } & value & 7 & 10 & 13 & 25 \\
\hline thickness of the object1 & 50 & 49 & 54 & 52 & 55 \\
\hline $\begin{array}{c}\text { distance between the front } \\
\text { surface of object 2 and } \\
\text { reference plane }\end{array}$ & 140 & 143 & 141 & 139 & 130 \\
\hline $\begin{array}{c}\text { diameter of the cylindrical } \\
\text { object }\end{array}$ & 70 & 73 & 74 & 72 & 77 \\
\hline radius of the object 3 & 95 & 102 & 97 & 97 & 109 \\
\hline
\end{tabular}

\section{CONCLUSIONS}

A major research issue associated with mobile service robots is the creation of autonomous environment sensing and recognition system for navigation and task execution that is robust and efficient. In this work, we proposed a new sensing algorithm based on the moire technique and stereo vision for acquiring more reliable range information and removing the $2 \pi$-ambiguity.

To see how the proposed algorithms can be applied to applications, we have done a series of experiments under various environmental conditions. The performed experiments show the feasibility of successful perception of the mobile robot with the proposed sensor algorithm on several environments.

\section{ACKNOWLEDGMENT}

This work was supported by the Ministry of Information \& Communications, Korea, under the Information Technology Research Center (ITRC) Support Program.

\section{REFERENCES}

[1] R.J. Valkenburg, A.M. Mcivor, Accurate 3D measurement using a structured light system, Image Vision Comput. 16, pp. 99-110, 1998.

[2] O. Faugeras, Three Dimensional Computer Vision: A Geometric Viewpoint, MIT Press, Cambridge, 1993.

[3] N. Ayache, Artificial Vision for Mobile Robots: Stereo Vision and Multisensory Perception, MIT Press, Cambridge, 1991.

[4] K.M. Lee, C.J. Kuo, Surface reconstruction from photometric stereo images, J. Opt. Soc. Am. A 10, pp. 855-867, 1993.

[5] Z. Zhang, O. Faugeras, A 3D world model builder with a mobile robot, Int. J. Robot. Res. 11, pp. 269-285, 1992

[6] P. Weckesser, R. Dillmann, Modeling unknown environments with a mobile robot, Robot. Auton. Syst. 23, pp. 293-300, 1998.

[7] H.R. Everett, Sensors for Mobile Robots: Theory and Application, A.K. Peters, Natick, 1995.

[8] G.N. DeSouza, A.C. Kak, Vision for mobile robot navigation: a survey, IEEE Trans. Pattern Anal. Mach. Vision 24, pp. 237-267, 2002.

[9] T. Kanade, Three-Dimensional Machine Vision, Kluwer Academic Publishers, Boston, 1989.

[10]J.E. Agapakis, J.M. Katz, J.M. Friedman, G.N. Epstein, Vision-aided robotic welding: an approach and a flexible implementation, Int. J. Robot. Res. 9. pp. 17-34, 1993.

[11]M. Suk, S.M. Bhandarkar, Three-Dimensional Object Recognition From Range Images, Springer-Verlag New York, Secaucus, 1992.

[12]M. Amann, T. Bosch, M. Lescure, R. Myllyla, M. Rioux, Laser ranging: a critical review of usual techniques for distance measurement, Opt. Eng. 40, pp. 10-19, 2001.

[13]Min Young Kim, Hyungsuck Cho, "An active trinocular vision system of sensing indoor navigation environment for mobile robots," Sensors and Actuators A: Physical, Vol. 125, pp. 192-209, 2006

[14]H. Takasaki, Moire Topography, Applied optics, vol.12, no.4, pp.845850,1973

[15]K.J. Gåsvik, Optical Metrology, 2nd edition, John Wiley \& Sons Ltd, 1995.

[16]J.F. Cardenas-Garcia, S. Zhang and F.Z. Shen, Implementation and use of an automated projection Moire experimental set-up, Optics and Lasers in Engineering, v. 21, pp. 77-98, 1994.

[17]Website http://repairfaq.ece.drexel.edu/sam/CORD/leot/course06_mod08/mod0608.html

[18]D. A. Forsyth and J. Ponce, Computer Vision: A Modern Approach, Prentice Hall, 2003.

[19]Zhang, "Flexible Camera Calibration by Viewing a Plane from Unknown Orientations," ICCV99, 1999 